Virginia Commonwealth University vCU Scholars Compass

2011

\title{
Impurity complexes and conductivity of Ga-doped $\mathrm{ZnO}$
}

D. O. Demchenko

Virginia Commonwealth University

B. Earles

Virginia Commonwealth University

H. Y.Liu

Virginia Commonwealth University

See next page for additional authors

Follow this and additional works at: http://scholarscompass.vcu.edu/phys_pubs

Part of the Physics Commons

Demchenko, D.O., Earles, B., Liu, H.Y., et al. Impurity complexes and conductivity of Ga-doped ZnO. Physical Review B, 84, 075201 (2011). Copyright (C) 2011 American Physical Society.

\section{Downloaded from}

http://scholarscompass.vcu.edu/phys_pubs/62

This Article is brought to you for free and open access by the Dept. of Physics at VCU Scholars Compass. It has been accepted for inclusion in Physics Publications by an authorized administrator of VCU Scholars Compass. For more information, please contact libcompass@vcu.edu. 
Authors

D. O. Demchenko, B. Earles, H. Y. Liu, Vitaliy Avrutin, N. Izyumskaya, Ü. Özgür, and Hadis Morkoç 


\title{
Impurity complexes and conductivity of Ga-doped $\mathrm{ZnO}$
}

\author{
D. O. Demchenko, ${ }^{1}$ B. Earles, ${ }^{1}$ H.Y. Liu, ${ }^{2}$ V. Avrutin, ${ }^{2}$ N. Izyumskaya, ${ }^{2}$ Ü. Özgür, ${ }^{2}$ and H. Morkoç ${ }^{1,2}$ \\ ${ }^{1}$ Department of Physics, Virginia Commonwealth University, Richmond, Virginia 23284, USA \\ ${ }^{2}$ Department of Electrical Engineering, Virginia Commonwealth University, Richmond, Virginia 23284, USA
}

(Received 7 February 2011; revised manuscript received 15 April 2011; published 1 August 2011)

\begin{abstract}
Using hybrid functional theory together with experimental measurements, we investigate the influence of gallium impurities and their complexes on electrical properties of $\mathrm{ZnO}$. In contrast to the behavior of isolated $\mathrm{Ga}$ impurities and native defects, the calculated formation energies of $\mathrm{Ga}$ complexes are consistent with our experimental data. We show that for high levels of Ga doping the acceptor behavior of $\mathrm{Ga}_{\mathrm{Zn}}-V_{\mathrm{Zn}}$ and $\mathrm{Ga}_{\mathrm{Zn}}-\mathrm{O}_{i}$ complexes explains the conductivity measurements and compensation levels in $\mathrm{ZnO}$. The computed binding energies of these complexes are also consistent with the binding energies obtained from the measurements of the temperature dependence of carrier mobility. Our results show that the formation of defect complexes, often overlooked by theory, can be indispensable in capturing the defect physics.
\end{abstract}

DOI: 10.1103/PhysRevB.84.075201

PACS number(s): 71.55.Gs, 71.15.Mb, 71.20.Nr

\section{INTRODUCTION}

Zinc oxide $(\mathrm{ZnO})$ is a material of practical interest showing significant advantages in several areas including: ultraviolet optoelectronic devices, transparent conducting oxide (TCO) thin films, and spintronics. ${ }^{1}$ A large band gap $(3.3 \mathrm{eV}$ at room temperature), large exciton binding energy $(60 \mathrm{meV}),{ }^{2}$ high electron mobility, visible transparency, and high emission efficiency also make $\mathrm{ZnO}$ attractive for light-emitting devices. These advantages have sparked interest in understanding the physics of defects and impurities in $\mathrm{ZnO}{ }^{3}$ However, due to known doping asymmetry, a stable $p$-type $\mathrm{ZnO}$ has been difficult to fabricate, ${ }^{4-7}$ delaying the development of $\mathrm{ZnO}$-based devices. On the other hand, $n$-type $\mathrm{ZnO}$ heavily doped with $\mathrm{Ga}^{8}$ has been suggested as a material with potential application as a transparent electrode in dye-sensitized solar cells,, 910 light-emitting devices, ${ }^{11}$ and transparent thin film transistors. ${ }^{12}$

The first principles calculations of defects and impurities ${ }^{13}$ using the local density approximation (LDA) and generalized gradient approximation (GGA) to the density functional theory (DFT) have been the subject of intense debate ${ }^{14,15}$ due to their notorious inconsistency, particularly in the case of $\mathrm{ZnO} .{ }^{16-24}$ The most widely studied is the case of oxygen vacancy in $\mathrm{ZnO}$, where the defect formation energies and the thermodynamic transition levels computed by different approaches within LDA/GGA vary by several electronvolts. ${ }^{25}$ The reason for such a large discrepancy lies in the variety of correction schemes to the LDA/GGA value of the band gap $\left(E_{g}=0.67 \mathrm{eV}\right.$ within LDA-DFT). Recently it has been demonstrated for a variety of defects in different materials that hybrid functional calculations can dramatically improve the accuracy of the computed defect formation energies. ${ }^{26-28}$ Presently, the hybrid functional approach provides a practical and affordable method for illuminating the defect physics. In its ability to reproduce the experimental band gaps, it is inferior only to the quasiparticle $G W$ method, ${ }^{29}$ which is very computationally expensive.

In this paper we demonstrate that by using hybrid functional calculations, we can reliably predict the formation properties of $\mathrm{Ga}$ impurities and their complexes in $\mathrm{ZnO}$. We calculate the formation energies as a function of the Fermi level of native defects in $\mathrm{ZnO}$, such as interstitial oxygen $\mathrm{O}_{i}, \mathrm{Zn}$ vacancies $V_{\mathrm{Zn}}$, as well as dopant $\mathrm{Ga}$ in the interstitial and substitutional sites, i.e. $\mathrm{Ga}_{i}$ and $\mathrm{Ga}_{\mathrm{Zn}}$. Due to both $\mathrm{Ga}_{i}$ and $\mathrm{Ga}_{\mathrm{Zn}}$ donor-like behavior, our calculations suggest that the Coulomb interactions between $\mathrm{Ga}$ and acceptor-like defects, such as $\mathrm{O}_{i}$ and $V_{\mathrm{Zn}}$, would lead to bound complexes. Furthermore, we show that these complexes, rather than isolated impurities, dominate the electrical properties of Ga-doped $\mathrm{ZnO}$. The calculated energetics of $\mathrm{Ga}_{\mathrm{Zn}}-V_{\mathrm{Zn}}$ and $\mathrm{Ga}_{\mathrm{Zn}}-\mathrm{O}_{i}$ complexes are consistent with Hall effect measurements in Ga-doped $\mathrm{ZnO}$ (GZO) samples grown on a-sapphire substrates by plasmaenhanced molecular beam epitaxy (PE MBE) under O-rich and O-deprived conditions.

\section{METHOD OF CALCULATION}

Specifically, our calculations are based on the HeydScuseria-Ernzerhof (HSE) ${ }^{30}$ hybrid functional implemented in the VASP code ${ }^{31}$ with the projector augmented wave (PAW) method. ${ }^{32,33}$ In a hybrid functional calculation, the local density exchange correlation part of the density functional is mixed with a Fock-type exchange part in varying proportions. In the HSE case, the Fock exchange interactions are separated into long- and short-range parts. The nonlocal Fock exchange potential is screened at long distances (similar to a screened exchange approach), ${ }^{34,35}$ and the long-ranged part is replaced with an approximate semilocal expression. It is also often useful to tune the amount of exact exchange for a particular material in order to obtain the best agreement of computed band gap with experiment, so-called $\alpha$-tuned hybrid functionals. We use the $\alpha$-tuned HSE hybrid functional, where the amount of exact exchange is set to be $37.5 \%$. This functional has been shown to most accurately reproduce both the band gap and the lattice properties, ${ }^{27}$ which are necessary for capturing the correct defect energetics. This approach has recently been used to describe the formation energies and thermodynamic transition levels of various defects in $\mathrm{ZnO}^{27}$ The screening parameter in HSE was kept at $0.2 \AA^{-1}$. The $3 d$ electrons of $\mathrm{Ga}$ are treated as the core electrons, while $\mathrm{Zn} 3 d, 4 s, 4 p$, and $\mathrm{O} 2 s, 2 p$ are considered as valence electrons. The resulting band gap of $3.43 \mathrm{eV}$ is in excellent agreement with the low-temperature experimental value. The calculated lattice parameters for wurtzite $\mathrm{ZnO} a=3.241 \AA$ and 
$c=5.258 \AA$ also agree with the experiment well, where $a=3.242 \AA$ and $c=5.188 \AA$. Supercells with 72 atoms in the unit were used. All atomic structures were relaxed to yield forces of $0.05 \mathrm{eV} / \AA$ or less. The resulting geometries of $\mathrm{Ga}_{\mathrm{Zn}}-V_{\mathrm{Zn}}$ and $\mathrm{Ga}_{\mathrm{Zn}}-\mathrm{O}_{i}$ complexes are shown in Fig. 1. Significant lattice distortions are evident, especially in the case of $\mathrm{Ga}_{\mathrm{Zn}}-\mathrm{O}_{i}$, where the Coulomb attraction reduces the $\mathrm{Ga}-\mathrm{O}$ bond length by $0.24 \AA$. The plane-wave basis sets with $400 \mathrm{eV}$ cutoff were used for electronic states calculations, while those with $300 \mathrm{eV}$ cutoff were used for atomic structure relaxations. It must be noted that both the structure relaxation and the electronic structure were computed using the HSE hybrid functional. We found that an efficient relaxation using LDA/GGA with subsequent HSE calculations of electronic structure can introduce a very large error in the formation energy, particularly in cases where shallow occupied defect levels are formed. This is due to the hybridization of the defect states with valence or conduction band within LDA/GGA, which leads to the wrong charge density and incorrect forces and relaxation. ${ }^{25}$ For example, in the case of the $\mathrm{Zn}$ vacancy $V_{\mathrm{Zn}}{ }^{0}, V_{\mathrm{Zn}}{ }^{1-}$, and $V_{\mathrm{Zn}}{ }^{2-}$, we compute this error to be $1.57,0.93$, and $0.24 \mathrm{eV}$, respectively. In all calculations $\Gamma$-point eigenvalues only were used. Due to the periodic boundary conditions, the spurious interactions between the defects leads to defect state dispersion, which in turn may lead to a relatively large error in the defect formation energy. ${ }^{13}$ In most cases considered here, this error is on the order of $0.1-0.2 \mathrm{eV}$ and can be as high as $0.34 \mathrm{eV}$ in the case of $V_{\mathrm{Zn}}{ }^{0}$. This error can be estimated by computing the HSE band structure and determining the occupied defect band weighted average, which gives a good approximation for the isolated defect level. The error in the formation energy was then corrected by using the difference between the defect band average and the eigenvalue at the $\Gamma$ point. Spin polarized calculations were performed in all cases, as many defects form half-filled localized states. The defect formation energies $E_{f}$ are defined in a standard way ${ }^{13}$

$$
E_{f}=E_{d}-E_{p}-\sum_{i} \Delta n_{i} \mu_{i}+q E_{F}+\Delta E_{M},
$$

where $E_{d}$ is the energy of the supercell containing defect, $E_{p}$ the energy of a pure nanocrystal, $\Delta n_{i}$ the difference in number of atoms of $i$ th kind, $\mu_{i}$ their atomic chemical potential, $q$ the charge of the defect, $E_{F}$ the Fermi energy (electrons are exchanged between a defect and the Fermi level), and $\Delta E_{M}$ the Makov-Payne ${ }^{36,37}$ energy correction for spurious interactions of charged defects due to the periodic boundary conditions. Here the Madelung energy was used, while dipole and higher-order interactions were deemed negligible. ${ }^{27}$ The chemical potentials depend on growth conditions and sources of impurities and can be estimated from HSE calculations and experimental formation enthalpies. To simulate experimental growth conditions, we assume that zinc and oxygen chemical potentials $\mu_{\mathrm{Zn}}$ and $\mu_{\mathrm{O}}$ depend on oxygen pressure in the growth system. In our calculations for the oxygen-rich limit, $\mu_{\mathrm{Zn}}=$ $\mu_{\mathrm{Zn} \text { (metal) }}+\Delta H_{\mathrm{ZnO}}$ and $\mu_{\mathrm{O}}=\mu_{\mathrm{O} 2} / 2$, and for the oxygen-poor limit, $\mu_{\mathrm{Zn}}=\mu_{\mathrm{Zn} \text { (metal) }}$ and $\mu_{\mathrm{O}}=\mu_{\mathrm{O} 2} / 2+\Delta H_{\mathrm{ZnO}}$, where $\Delta H_{\mathrm{ZnO}}$ is the bulk $\mathrm{ZnO}$ enthalpy of formation. The HSE hybrid functional yields $\Delta H_{\mathrm{ZnO}}=-3.32 \mathrm{eV}$ (in experiment
$\left.\Delta H_{\mathrm{ZnO}}=-3.63 \mathrm{eV}\right)$. HSE values of total energy for bulk metallic $\mathrm{Zn}$ yields $\mu_{\mathrm{Zn} \text { (metal) }}=-2.37 \mathrm{eV}$, and that of oxygen molecule $\mu_{\mathrm{O} 2}=-20.66 \mathrm{eV}$. The source of gallium is assumed to be metallic stable orthorhombic phase, for which we obtain $\mu_{\mathrm{Ga}}=3.75 \mathrm{eV}$.

From the experimental point of view, O-rich (or Zn-rich) conditions imply that the supply of reactive $\mathrm{O}$ atoms exceeds that of $\mathrm{Zn}$ atoms (or vice versa). The partial pressures of reactive $\mathrm{O}$ or $\mathrm{Zn}$ can be estimated from the growth rate $(\sim 1.5 \mathrm{~nm} / \mathrm{min}$ in our case) at $1: 1$ reactive oxygen-to-metal flux ratio (near-stoichiometric growth conditions), determined from the dependence of growth rate on oxygen pressure at constant $\mathrm{Zn}$ flux and taking into account the incorporation efficiency of $\sim 1 / 3$ of $\mathrm{Zn}$ atoms into the $\mathrm{ZnO}$ lattice during plasma-assisted MBE on sapphire ${ }^{38}$. Assuming $\mathrm{Zn}$ arrives as a monoatomic reagent, its partial pressure above the $\mathrm{ZnO}$ growing surface was estimated to be between $6 \times 10^{-7}$ and $1 \times$ $10^{-6}$ Torr. The reaction rate between $\mathrm{Zn}$ and molecular $\mathrm{O}_{2}$ at the growth temperatures is very low; therefore, plasma source is used to generate monoatomic reactive oxygen. The 1:1 ratio of atomic $\mathrm{O}$ to $\mathrm{Zn}$ is obtained at the total oxygen pressure of 8 to $10 \times 10^{-6}$ Torr in the growth chamber measured with a vacuum gauge, while the estimated partial pressure of reactive oxygen is slightly below $1 \times 10^{-6}$ Torr. Assuming the flux of reactive components varies linearly with their partial pressure, the partial pressure of reactive oxygen can be estimated to be $5 \times 10^{-7}$ Torr for Zn-rich conditions (the total oxygen pressure is $\sim 4$ to $5 \times 10^{-6}$ Torr) and around $3 \times$ $10^{-6}$ Torr for oxygen-rich conditions (the total oxygen pressure is $1.5 \times 10^{-5}$ Torr). This pressure difference is four orders of magnitude larger than the equilibrium oxygen partial pressure over $\mathrm{ZnO}\left(1.75 \times 10^{-10}\right.$ Torr at growth temperature, deduced from the data in Ref. 39), illustrating that experimentally O-rich and O-poor conditions are significantly far apart. These experimental conditions of the plasma enhanced MBE growth are away from thermal equilibrium. Nevertheless, the computed defect formation energies are still physically meaningful, allowing comparison of the theory and the experiment. ${ }^{13}$ Nonequilibrium growth conditions imply that a higher energy defect state can form with concentrations exceeding their equilibrium values. However, defects with high formation energy will always be more difficult to create, since high energy always needs to be spent in order to form them. Since it is difficult to determine the exact correspondence of the partial pressures of reagents to the changes of the chemical potentials during a nonequilibrium growth, we can expect to capture trends between the two limiting growth conditions.

\section{RESULTS AND DISCUSSION}

$\mathrm{Ga}$ is known to be a shallow donor, substituting for $\mathrm{Zn}$ in the $\mathrm{ZnO}$ lattice (Fig. 1), while interstitial Ga impurities have much larger formation energies. We have tested octahedral, tetrahedral, and two inequivalent hexagonal sites in the $\mathrm{ZnO}$ wurtzite lattice as possible sites for $\mathrm{Ga}_{i}$. The formation energies for $\mathrm{Ga}_{i}$ at these sites differ by a maximum of $0.3 \mathrm{eV}$, while all of them are much higher than substitutional Gazn. For instance, this difference for neutral impurities is $7.88 \mathrm{eV}$. This implies negligible equilibrium concentrations of the interstitial 
(a)

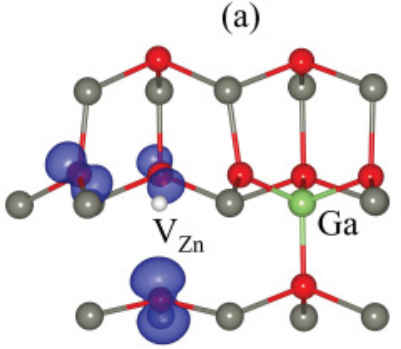

(b)

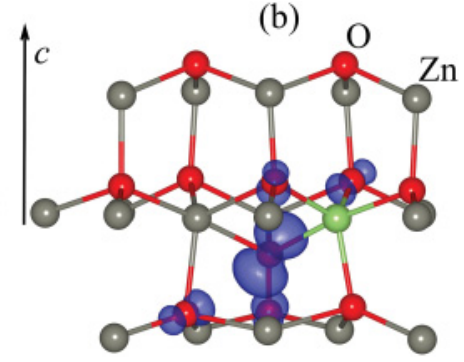

FIG. 1. (Color online) (a) Charge densities of the electron trapped at the $\left(\mathrm{Ga}_{\mathrm{Zn}}-V_{\mathrm{Zn}}\right)^{1-}$ complex and (b) $\left(\mathrm{Ga}_{\mathrm{Zn}}-\mathrm{O}_{i}\right)^{2-}$ complex. The isosurfaces are taken at $6 \%$ of the maximum. Both complexes are deep acceptors with low formation energy in the O-rich environment. $\left(\mathrm{Ga}_{\mathrm{Zn}}-\mathrm{O}_{i}\right)^{2-}$ forms three nearly degenerate defect states of the same symmetry (one shown).

$\mathrm{Ga}$ impurities. Previous theoretical studies of $\mathrm{Ga}_{\mathrm{Zn}}$ in $\mathrm{ZnO}$ often were concentrated on $\mathrm{Ga}$ as a codopant (with $\mathrm{N})^{40-42}$ in order to circumvent the doping asymmetry and obtain a stable $p$-type $\mathrm{ZnO}$ and (with $\mathrm{Co})^{43}$ to fabricate $\mathrm{ZnO}$ as a dilute magnetic semiconductor. First principles DFT calculations of an isolated $\mathrm{Ga}_{\mathrm{Zn}}$ impurity yielded a donor thermodynamic transition level at $1.5 \mathrm{eV}$ above the valence band maximum (VBM) using GGA, around $2.1 \mathrm{eV}$ using GGA $+U$ (for $U=$ $0.5 \mathrm{eV}$ ), and $3.9 \mathrm{eV}$ by using band gap extrapolation formula (introduced in Ref. 20). ${ }^{44}$ We find $\mathrm{Ga}$ zn to be a shallow donor with $+/ 0$ thermodynamic transition level being at $3.00 \mathrm{eV}$ above the VBM (Fig. 2). The formation energy of $\mathrm{Ga}_{\mathrm{Zn}}{ }^{0}$ is $-4.02 \mathrm{eV}$ in the O-rich conditions, and $-0.67 \mathrm{eV}$ in the $\mathrm{O}$-poor conditions. This implies that O-rich conditions would produce much more abundant numbers of Ga donors in comparison with $\mathrm{O}$-poor conditions. In fact, the experimental picture is the exact opposite. Measured carrier concentrations in samples grown under O-poor conditions are several times to more than one order of magnitude higher than those in

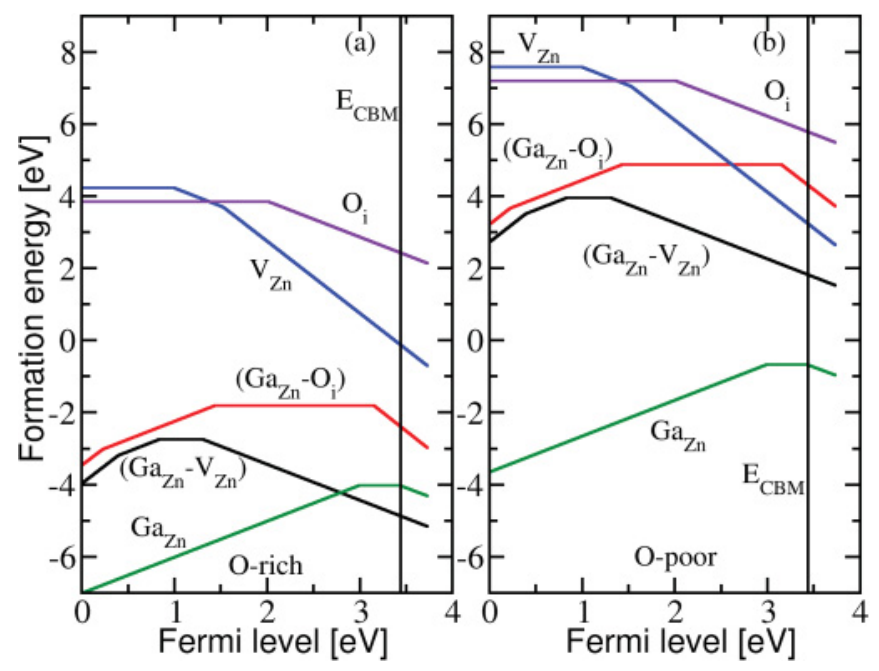

FIG. 2. (Color online) Formation energies of defects as a function of the location of Fermi energy (origin is set at the VBM) in (a) oxygen rich and (b) oxygen poor environment. Positive (negative) slope corresponds to positive (negative) defect charge state. The ends of the formation energy lines correspond to the experimental $E_{F}$ of Ga heavily doped $\mathrm{ZnO}, 0.3 \mathrm{eV}$ above the $\mathrm{CBM}$ (vertical line).

samples grown under O-rich conditions. ${ }^{45-47}$ In particular, the measured carrier concentrations in our GZO layers grown by $\mathrm{PE} \mathrm{MBE}$ at $\mathrm{P}_{\mathrm{O} 2}=4.5 \times 10^{-6}$ Torr (metal-to-reactive oxygen ratio $<1^{8}$ ) are $\sim 5$ to $8 \times 10^{20} \mathrm{~cm}^{-3}$, while those in the layers grown at $\mathrm{P}_{\mathrm{O} 2}=1.5 \times 10^{-5}$ Torr (metal-to-reactive oxygen ratio $>1^{8}$ ) are $2 \times 10^{19} \mathrm{~cm}^{-3}$.

Acceptor-like defects, such as interstitial oxygen $\mathrm{O}_{i}$ and zinc vacancy $V_{\mathrm{Zn}}$ considered here, have relatively high formation energies (Fig. 2). For both O-rich and O-poor growth regimes at the experimental Fermi levels of approximately $0.3 \mathrm{eV}$ above the conduction band minimum (CBM), the formation energy of $V_{\mathrm{Zn}}\left(\mathrm{O}_{i}\right)$ acceptor is $3.63 \mathrm{eV}(6.48 \mathrm{eV})$ higher than that of the $\mathrm{Ga}_{\mathrm{Zn}}$ donor. Therefore, regardless of the growth conditions, the formation energies of these acceptor defects do not suggest any appreciable compensation. Nevertheless, in the samples grown under O-rich conditions, temperature-dependent mobility measurements suggest $94 \%$ compensation in as-grown samples (reduced to $66 \%$ upon thermal annealing), while the compensation in the layers grown under O-poor conditions ranges from 20 to $30 \%$. Therefore, other mechanisms of compensation must be present.

In order to resolve these inconsistencies, here we suggest that both the compensation mechanism and the conductivity measurements can be explained by involving defect complexes. Due to large concentrations of $\mathrm{Ga}$ in our samples the natural candidates for such complexes are the $\mathrm{Ga}_{\mathrm{Zn}}\left(\right.$ or $\left.\mathrm{Ga}_{i}\right)$ donor, $V_{\mathrm{Zn}}\left(\right.$ or $\mathrm{O}_{i}$ ) acceptor pairs. Indeed, our hybrid functional calculations show that both the $\mathrm{Ga}_{\mathrm{Zn}}-V_{\mathrm{Zn}}$ and $\mathrm{Ga}_{\mathrm{Zn}}-\mathrm{O}_{i}$ complexes are deep acceptors with $0 /-$ thermodynamic transition levels at $1.31 \mathrm{eV}$ and $3.15 \mathrm{eV}$ above $\mathrm{VBM}$, respectively. The $\mathrm{Ga}_{i}-V_{\mathrm{Zn}}$ complex does not form with $\mathrm{Ga}_{i}$ moving into the $\mathrm{Zn}$ vacancy as a result of atomic relaxation. The defect state charge densities for $\mathrm{Ga}_{\mathrm{Zn}}-V_{\mathrm{Zn}}$ and $\mathrm{Ga}_{\mathrm{Zn}}-\mathrm{O}_{i}$ complexes are shown in Fig. 1, where significant lattice relaxation is evident, suggesting the expected strong acceptor-donor Coulomb interactions. The defect states are derived from the oxygen $p$-states localized at the acceptor's neighboring $\mathrm{O}$ atoms. Thus, in both cases, these acceptor complexes trap the electrons at nearby oxygen sites, while the Gazn plays a stabilizing role, dramatically reducing the acceptor formation energies.

In the O-rich environment [Fig. 2(a)], for $E_{F}$ beyond $2.8 \mathrm{eV}$, the formation energy of the $\mathrm{Ga}_{\mathrm{Zn}}-V_{\mathrm{Zn}}$ complex is significantly lower than that of the $\mathrm{Ga}_{\mathrm{Zn}}$. For example, in the most extreme case of $E_{F}$ at $0.3 \mathrm{eV}$ in the conduction band, this difference reaches $0.85 \mathrm{eV}$, suggesting that this acceptor is responsible for the observed compensation ratios. Isolated zinc vacancies have been shown to be mobile well below room temperature and readily binding into complexes with other defects. ${ }^{48}$ Therefore, the $G a_{Z n}-V_{Z n}$ complexes can efficiently form during the cooldown period after growth. The $\mathrm{Ga}_{\mathrm{Zn}}-\mathrm{O}_{i}$ complex has formation energy that is $1.35 \mathrm{eV}$ higher, and therefore its contribution is less significant.

In the O-poor environment [Fig. 2(b)], the picture is dramatically different. While the formation energies of the $\mathrm{Ga}_{\mathrm{Zn}}$ donor (and $V_{\mathrm{Zn}}, \mathrm{O}_{i}$ acceptors) increase by $3.35 \mathrm{eV}$ due to the differences in $\mathrm{Zn}$ chemical potential, the formation energies of $\mathrm{Ga}_{\mathrm{Zn}}-V_{\mathrm{Zn}}$ and $\mathrm{Ga}_{\mathrm{Zn}}-\mathrm{O}_{i}$ complexes increase by $6.70 \mathrm{eV}$. In this case, regardless of the Fermi level location, the formation energies of these complexes are, respectively, 2.48 and $4.69 \mathrm{eV}$ 


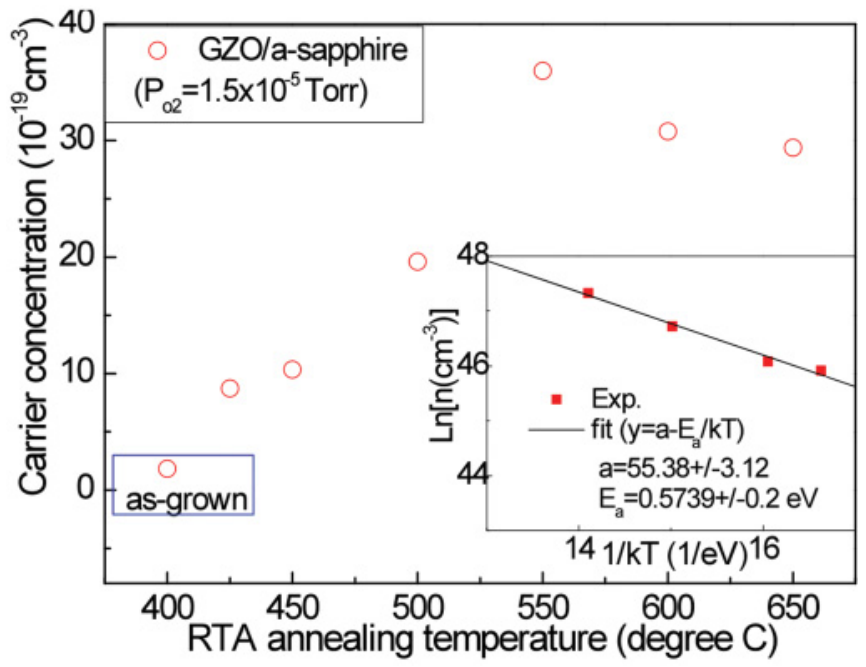

FIG. 3. (Color online) Determining the binding energy of the defect complexes for sample grown in the O-rich environment based on measurements of the temperature-dependent carrier concentrations. The concentrations are plotted in the Arrhenius coordinates, yielding an activation energy $E_{a}=0.57 \mathrm{eV}$.

higher than that of the $\mathrm{Ga}_{\mathrm{Zn}}$ donor, and no compensation via these complexes is expected.

These computed defect energetics are in agreement with our experimental data obtained for the GZO layers grown on a-plane sapphire at the same substrate temperature of $400{ }^{\circ} \mathrm{C}$ using the same $\mathrm{Ga}$ and $\mathrm{Zn}$ fluxes but under $\mathrm{O}$-poor condition $\left(\mathrm{P}_{\mathrm{O} 2}=4.5 \times 10^{-6}\right.$ Torr $)$ and O-rich condition $\left(\mathrm{P}_{\mathrm{O} 2}=1.5 \times 10^{-5}\right.$ Torr $)$. Detailed information on the growth conditions and the effects of rapid thermal annealing (RTA) on the electrical properties of the samples can be found elsewhere. ${ }^{8}$ The resistivities of the layers grown under O-rich and in O-poor conditions are $\sim 4.5 \times 10^{-2}$ and $\sim 2.8 \times$ $10^{-4} \Omega \mathrm{cm}$, respectively. This is consistent with the results of our hybrid functional calculations predicting a much more efficient formation of acceptor defect complexes under oxygen-rich conditions, providing a compensation mechanism and decreasing the electric conductivity.

We estimate the binding energies of $\mathrm{Ga}_{\mathrm{Zn}}-V_{\mathrm{Zn}}$ and $\mathrm{Ga}_{\mathrm{Zn}}-\mathrm{O}_{i}$ complexes as the formation energy difference of a complex and its constituents. In our O-rich grown samples, the carrier concentration is $2.0 \times 10^{19} \mathrm{~cm}^{-3}$, placing the Fermi level approximately at $0.1 \mathrm{eV}$ below the CBM. In this case, the $\mathrm{Ga}_{\mathrm{Zn}}-V_{\mathrm{Zn}}$ binding energy is $0.75 \mathrm{eV}$, and that of $\mathrm{Ga}_{\mathrm{Zn}}-\mathrm{O}_{i}$ is $0.66 \mathrm{eV}$. These binding energies are in reasonable agreement with the measured value of $0.57 \mathrm{eV}$, obtained from the temperature-dependent concentrations shown in Fig. 3. Upon the RTA treatment in the temperature range from 400 to $550{ }^{\circ} \mathrm{C}$, resistivity of the samples grown under O-rich conditions reduced to $\sim 5.7 \times 10^{-4} \Omega \mathrm{cm}$, and the compensation ratio calculated by the method reported by Look et al. ${ }^{49}$ decreased from $\sim 94$ to $\sim 66 \%$. The reduction in the resistivity upon annealing is mainly due to the increases in carrier concentration (shown in Fig. 3), while mobilities in the annealed samples slightly varied around $20 \mathrm{~cm}^{2} / \mathrm{Vs}$. The increase in carrier concentration of the annealed GZO layers is likely due to the dissociation of $\mathrm{Ga}_{\mathrm{Zn}}-V_{\mathrm{Zn}}$ and/or $\mathrm{Ga}_{\mathrm{Zn}}-\mathrm{O}_{i}$ complexes. The carrier concentration in the samples annealed in the temperature range from 425 to $550{ }^{\circ} \mathrm{C}$ follows the Arrhenius law (see the inset in Fig. 3), and binding energy of defect complexes was found to be $\sim 0.57 \pm 0.2 \mathrm{eV}$. (Further increase in annealing temperature from 550 to $650{ }^{\circ} \mathrm{C}$ leads to the gradual decrease in the carrier concentrations in all samples, likely due to the decrease in structural quality of the material.)

\section{CONCLUSIONS}

In summary, we have shown through hybrid functional calculations that inclusion of impurity complexes is crucial for understanding the electric properties of heavily doped $\mathrm{ZnO}$. We find that the measured conductivity data cannot be explained by the isolated impurities alone. On the other hand, the computed electronic properties of impurity complexes provide a picture, which is in agreement with the measured conductivity, compensation ratios, and binding energies. In the O-rich environment, the formation energies of the acceptor $\mathrm{Ga}_{\mathrm{Zn}}-V_{\mathrm{Zn}}$ and $\mathrm{Ga}_{\mathrm{Zn}}-\mathrm{O}_{i}$ complexes are significantly lower than that of the donor $\mathrm{Ga}_{\mathrm{Zn}}$. This provides an efficient mechanism for experimentally observed high compensation ratios and lower conductivity. In the O-poor environment, the complex formation energies are $6.70 \mathrm{eV}$ higher, indicating that in this case these complexes do not form, which leads to much larger values of carrier concentrations and conductivity.

\section{ACKNOWLEDGMENTS}

This paper used computational facilities of the VCU Center for High Performance Computing. We thank M. Reshchikov for useful discussions. The work was partially supported by DOE (Project No. DE-FG-02-08ER46547) through University of Wisconsin.
${ }^{1}$ H. Morkoç and Ü. Özgür, Zinc Oxide: Fundamentals, Materials and Device Technology, (Wiley-VCH, Weinheim, 2009).

${ }^{2}$ U. Ozgur, Y. I. Alivov, C. Liu, A. Teke, M. A. Reshchikov, S. Dogan, V. Avrutin, S. J. Cho, and H. Morkoç, J. Appl. Phys. 98, 041301 (2005).

${ }^{3}$ M. D. McCluskey and S. J. Jokela, J. Appl. Phys. 106, 071101 (2009).

${ }^{4}$ C. H. Park, S. B. Zhang, and S. -H. Wei, Phys. Rev. B 66, 073202 (2002).
${ }^{5}$ V. Avrutin, D. J. Silversmith, H. Morkoç, Proc. IEEE 98, 1269 (2010).

${ }^{6}$ S. B. Zhang, J. Phys. Condens. Matter 14, R881 (2002).

${ }^{7}$ S. H. Wei, Comput. Mater. Sci. 30, 337 (2004).

${ }^{8}$ H. Y. Liu, V. Avrutin, N. Izyumskaya, M. A. Reshchikov, Ü. Özgür, and H. Morkoç, Phys. Status Solidi RRL 4, 70 (2010).

${ }^{9}$ H. H. Chen, A. D. Pasquier, G. Saraf, J. Zhong, and Y. C. Lu, Semicond. Sci. Technol. 23, 045004 (2008). 
${ }^{10}$ A. de Souza Gonçalves, M. R. Davolos, N. Masaki, S. Yanagida, A. Morandeira, J. R. Durrant, J. N. Freitas, and A. F. Nogueira, Dalton Trans. 1487 (2008).

${ }^{11}$ Z. Y. Li, L. Gan, C. Z. Zhou, C. Wang, R. J. Liu, and D. Z. Zhang, Phys. Status Solidi A 207, 1993 (2010).

${ }^{12}$ P. F. Carcia, R. S. McLean, M. H. Reilly, and G. Nunes, Appl. Phys. Lett. 82, 1117 (2003).

${ }^{13}$ C. G. Van de Walle and J. Neugebauer, J. Appl. Phys. 95, 3851 (2004).

${ }^{14}$ P. A. Schultz, Phys. Rev. Lett. 96, 246401 (2006).

${ }^{15}$ B. R. Tuttle and S. T. Pantelides, Phys. Rev. Lett. 101, 089701 (2008).

${ }^{16}$ S. Lany, Y. J. Zhao, C. Persson, and A. Zunger, Appl. Phys. Lett. 86, 042109 (2005).

${ }^{17}$ F. Oba, S. R. Nishitani, S. Isotani, H. Adachi, and I. Tanaka, J. Appl. Phys. 90, 824 (2001).

${ }^{18}$ J. L. Zhao, W. Zhang, X. M. Li, J. W. Feng, and X. Shi, J. Phys. Condens. Matter 18, 1495 (2006).

${ }^{19}$ T. R. Paudel and W. R. L. Lambrecht, Phys. Rev. B 77, 205202 (2008).

${ }^{20}$ A. Janotti and C. G. Van de Walle, Phys. Rev. B 76, 165202 (2007).

${ }^{21}$ S. Lany and A. Zunger, Phys. Rev. B 72, 035215 (2005).

${ }^{22}$ A. F. Kohan, G. Cedar, D. Morgan, and C. G. Van de Walle, Phys. Rev. B 61, 15019 (2000).

${ }^{23}$ J. A. Chan, S. Lany, and A. Zunger, Phys. Rev. Lett. 103, 016404 (2009).

${ }^{24}$ S. Lany and A. Zunger, Phys. Rev. Lett. 98, 045501 (2007).

${ }^{25}$ S. Lany and A. Zunger, Phys. Rev. B 78, 235104 (2008).

${ }^{26}$ J. L. Lyons, A. Janotti, and C. G. Van de Walle, Appl. Phys. Lett. 97, 152108 (2010).

${ }^{27}$ F. Oba, A. Togo, I. Tanaka, J. Paier, and G. Kresse, Phys. Rev. B 77, 245202 (2008).

${ }^{28}$ J. L. Lyons, A. Janotti, and C. G. Van de Walle, Appl. Phys. Lett. 95, 252105 (2009)

${ }^{29}$ S. Lany and A. Zunger, Phys. Rev. B 81, 113201 (2010).
${ }^{30}$ J. Heyd, G. E. Scuseria, and M. Ernzerhof, J. Chem. Phys. 118, 8207 (2003).

${ }^{31}$ G. Kresse and J. Furthmüller, Phys. Rev. B 54, 11169 (1996).

${ }^{32}$ P. E. Blöchl, Phys. Rev. B 50, 17953 (1994).

${ }^{33}$ G. Kresse and D. Joubert, Phys. Rev. B 59, 1758 (1999).

${ }^{34}$ A. Seidl, A. Görling, P. Vogl, J. A. Majewski, and M. Levy, Phys. Rev. B 53, 3764 (1996).

${ }^{35}$ S. J. Clark, J. Robertson, S. Lany, and A. Zunger, Phys. Rev. B 81, 115311 (2010).

${ }^{36}$ G. Makov and M. C. Payne, Phys. Rev. B 51, 4014 (1995).

${ }^{37}$ M. Leslie and M. J. Gillan, J. Phys. C 18, 973 (1985).

${ }^{38}$ H. J. Ko, T. Yao, Y. Chen, and S. K. Hong, J. Appl. Phys. 92, 2354 (2002).

${ }^{39}$ D. F. Anthrop and A. W. Searcy, J. Phys. Chem. 68, 2335 (1964).

${ }^{40}$ X. Y. Duan, Y. J. Zhao, and R. H. Yao, Solid State Commun. 147, 194 (2008).

${ }^{41}$ X. M. Duan, C. Stampfl, M. M. M. Bilek, and D. R. McKenzie, Phys. Rev. B 79, 235208 (2009).

${ }^{42}$ S. Lany and A. Zunger, Phys. Rev. B 81, 205209 (2010).

${ }^{43}$ Y. He, P. Sharma, K. Biswas, E. Z. Liu, N. Ohtsu, A. Inoue, Y. Inada, M. Nomura, J. S. Tse, S. Yin, and J. Z. Jiang, Phys. Rev. B 78, 155202 (2008).

${ }^{44}$ G. Y. Huang, C. Y. Wang, and J. T. Wang, Physica B 405, 158 (2010).

${ }^{45}$ S. Shirakata, T. Sakemi, K. Awai, and T. Yamamoto, Superlattices Microstruct. 39, 218 (2006).

${ }^{46}$ L. M. Wong, S. Y. Chiam, J. Q. Huang, S. J. Wang, J. S. Pan, and W. K. Chim, Appl. Phys. Lett. 98, 022106 (2011).

${ }^{47}$ B. Z. Dong, H. Hu, G. J. Fang, X. Z. Zhao, D. Y. Zheng, and Y. P. Sun, J. Appl. Phys. 103, 073711 (2008).

${ }^{48}$ F. Tuomisto, K. Saarinen, D. C. Look, and G. C. Farlow, Phys. Rev. B 72, 085206 (2005).

${ }^{49}$ D. C. Look, K. D. Leedy, D. H. Tomich, and B. Bayraktaroglu, Appl. Phys. Lett. 96, 062102 (2010). 